Supporting Information

For

\title{
The Ligand Trans Influence Governs Conformation in Cobalamin-Dependent Methionine Synthase
}

Angela S. Fleischhacker and Rowena G. Matthews

\section{Derivatization of equation 3}

The spectral signal is the fraction of MetH-off:

$$
\begin{aligned}
& \mathrm{Y}_{\mathrm{t}}=\frac{[\mathrm{MetH}-\mathrm{off}]+[\mathrm{MetH}-\text { off } \bullet \text { Fld }]}{[\mathrm{E}]_{\mathrm{t}}} \\
& {[\mathrm{E}]_{\mathrm{t}}=[\mathrm{MetH}-\mathrm{off}]+[\mathrm{MetH}-\text { off } \bullet \text { Fld }]+[\mathrm{MetH}-\text { on }]+[\mathrm{MetH}-\text { on } \bullet \text { Fld }]} \\
& \mathrm{Y}_{\mathrm{t}}=\frac{[\mathrm{MetH}-\text { off }]+[\mathrm{MetH}-\text { off } \bullet \text { Fld }]}{[\text { MetH }- \text { off }]+[\mathrm{MetH}-\text { off } \bullet \text { Fld }]+[\text { MetH }- \text { on }]+[\mathrm{MetH}-\text { on } \bullet \text { Fld }]}
\end{aligned}
$$

From the thermodynamic box in Figure 2 of the text, we define:

$$
\begin{aligned}
& \mathrm{K}_{\mathrm{a}}=\frac{[\mathrm{MetH}-\text { off } \bullet \text { Fld }]}{[\mathrm{MetH}-\text { off }][\text { Fld }]} \Rightarrow[\mathrm{MetH}-\text { off } \bullet \text { Fld }]=\mathrm{K}_{\mathrm{a}}[\mathrm{MetH}-\text { off }][\text { Fld }] \\
& \left(\mathrm{K}_{\mathrm{a}} / \mathrm{n}\right)=\frac{[\mathrm{MetH}-\text { on } \bullet \text { Fld }]}{[\mathrm{MetH}-\text { on }][\text { Fld }]} \Rightarrow[\mathrm{MetH}-\text { on } \bullet \text { Fld }]=\left(\mathrm{K}_{\mathrm{a}} / \mathrm{n}\right)[\mathrm{MetH}-\text { on }][\text { Fld }] \\
& \mathrm{K}_{\text {off } / \mathrm{on}}=\frac{[\mathrm{MetH}-\text { off }]}{[\mathrm{MetH}-\text { on }]} \Rightarrow[\mathrm{MetH}-\text { on }]=\frac{[\mathrm{MetH}-\text { off }]}{\mathrm{K}_{\text {off } / \text { on }}}
\end{aligned}
$$

Substituting equations S.3 and S.4 into equation S.2: 


$$
\begin{aligned}
& \mathrm{Y}_{\mathrm{t}}=\frac{[\mathrm{MetH}-\text { off }]+\mathrm{K}_{\mathrm{a}}[\mathrm{MetH}-\text { off }][\text { Fld }]}{[\text { MetH }- \text { off }]+\mathrm{K}_{\mathrm{a}}[\mathrm{MetH}-\text { off }][\text { Fld }]+[\mathrm{MetH}-\text { on }]+\left(\mathrm{K}_{\mathrm{a}} / \mathrm{n}\right)[\mathrm{MetH}-\text { on }][\text { Fld }]} \\
& \mathrm{Y}_{\mathrm{t}}=\frac{[\mathrm{MetH}-\text { off }]\left\{1+\mathrm{K}_{\mathrm{a}}[\text { Fld }]\right\}}{[\text { MetH }- \text { off }]\left\{1+\mathrm{K}_{\mathrm{a}}[\text { Fld }]\right\}+[\mathrm{MetH}-\text { on }]\left\{1+\left(\mathrm{K}_{\mathrm{a}} / \mathrm{n}\right)[\text { Fld }]\right\}}
\end{aligned}
$$

Substituting equation S.5 into equation S.7:

$$
\mathrm{Y}_{\mathrm{t}}=\frac{[\text { MetH }- \text { off }]\left\{1+\mathrm{K}_{\mathrm{a}}[\text { Fld }]\right\}}{[\text { MetH }- \text { off }]\left\{1+\mathrm{K}_{\mathrm{a}}[\text { Fld }]\right\}+\left\{\frac{[\text { MetH }- \text { off }]}{\mathrm{K}_{\text {off } / \text { on }}}\right\}\left\{1+\left(\mathrm{K}_{\mathrm{a}} / \mathrm{n}\right)[\text { Fld }]\right\}}
$$

We divide by [MetH-off] to arrive at equation 3 from the text:

$$
Y_{t}=\frac{1+K_{a}[\text { Fld }]}{\left(1+K_{a}[\text { Fld }]\right)+\left(1 / K_{\text {off } / n}\right)\left(1+\left(K_{a} / n\right)[\text { Fld }]\right)}
$$

